\title{
Reflexiones sobre la educación presencial universitaria durante la cuarentena por COVID-19
}

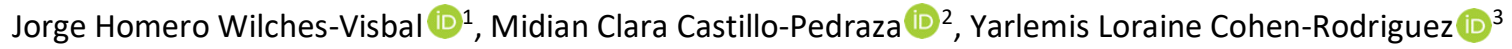

1. Universidad del Magdalena. Santa Marta, Colombia. Correo: jhwilchev@gmail.com - https://orcid.org/0000-0003-3649-5079

2. Universidad del Magdalena. Santa Marta, Colombia. Correo: midianclar@gmail.com - https://orcid.org/0000-0003-3170-3959

3. Universidad del Magdalena. Santa Marta, Colombia. Correo: yarlecohen@gmail.com - https://orcid.org/0000-0002-1889-313X

Señor Editor:

La educación es uno de los factores fundamentales para el progreso económico y social de cualquier civilización ${ }^{1-3}$. Algunos estudios han estimado que cada año de escolaridad adicional aumenta, en promedio, el producto interno bruto (PIB) de un país en un $0,37 \%{ }^{4} \mathrm{y}$ entre un $6-10 \%$ la productividad (e ingresos) de un individuo ${ }^{5}$. También se ha establecido que un aumento del $10 \%$ en el número de universidades per cápita de una región le representaría un PIB futuro per cápita 0,4\% mayor 6 . Asimismo, la educación promueve la movilidad social, los principios democráticos y disminuye la propensión a la criminalidad ${ }^{2,4,6}$.

Aunque la educación presencial es la más extendida y confiable, el creciente acceso a las tecnologías de la información y la comunicación (TIC), el encarecimiento de la educación presencial, las limitaciones de acceso físico a las instituciones y el carácter innovador de la formación no-presencial ${ }^{9}$, han propiciado el crecimiento sostenible de la modalidad virtual en los últimos años ${ }^{10}$.

La pandemia del nuevo coronavirus, denominada COVID-19, es una enfermedad infecciosa de las vías respiratorias ${ }^{11}$ que ha causado pérdidas económicas y sociales irreparables. Entre diciembre de 2019 y mediados de mayo de 2020, la COVID-19 se ha extendido a más de 200 países, con un saldo aproximado de 4.200 .000 infectados y 300.000 muertos. En Colombia, los infectados y fallecidos, en ese mismo periodo, rondan los 13000 y 500, respectivamente ${ }^{12}$.

Debido a la prolongada cuarentena, la COVID-19 ha representado una amenaza para la educación presencial global, ya que, por esta, cerca del $80 \%$ de niños y adolescentes del mundo dejaron de asistir a las escuelas en marzo pasado. Colateralmente, la cuarentena por COVID-19 ha causado trastornos en el proceso de aprendizaje, estrés psicológico en padres de familia, estudiantes y docentes, aumento en la tasa de deserción de estudiantes y pérdida de una ración importante de alimentos para muchos estudiantes de condición vulnerable ${ }^{13,14}$.

Para minimizar el detrimento, el estado y las universidades colombianas han realizado esfuerzos económicos y pedagógicos ingentes. En efecto, docentes de programas presenciales han experimentado, en poco tiempo, diversos desafíos de resiliencia a la educación no-presencial relacionados con: el poco dominio de las TICs; el tiempo disponible para preparar la clase, exacerbado en los que ministran más de una materia o lo hacen por primera vez; transmitir conocimientos prácticos relacionados con laboratorios y abordajes clínicos (punto álgido en docentes de carreras de salud); encontrar estrategias efectivas de flexibilización del aprendizaje que mantengan vinculados a los estudiantes con el proceso formativo y así atenuar la deserción; el solapamiento continuo de la vida personal y laboral; incertidumbre acerca del futuro laboral inmediato; compartir el mismo espacio de 
trabajo con los familiares sin afectar el rendimiento laboral de cada uno, entre otros.

En consecuencia, los docentes de educación presencial se han visto en la necesidad diaria de elaborar videoclases, clases en vivo, laboratorios virtuales, discusión de casos clínicos y videos de procedimientos clínicos. En la pandemia, Youtube, Whatsapp, Blackboard, Microsoft Teams, Zoom, OBS Studio y demás plataformas tecnológicas se tornaron de uso rutinario.

Estudiantes también han afrontado un sinnúmero de desafíos: adquirir equipos de cómputo o celulares y/o recarga de datos para asistir a las clases; responder a las exigencias diarias de las materias mientras se deparan con quehaceres domésticos y problemas de convivencia; mantener la regularidad y constancia en el proceso de aprendizaje sobre todo en lugares con limitado acceso tecnológico y/o constantes fallas del fluido eléctrico; lidiar con la incertidumbre de la nota final y de cómo pagarán el próximo semestre, entre otros. Particular preocupación para docentes y estudiantes de ciencias de la salud representan las materias clínicas ya que, en su mayoría, son insustituibles por escenarios virtuales.

En todas las universidades colombianas, el personal administrativo y docente ha asumido la tarea titánica de adaptarse eficientemente al reto de la no-presencialidad. En particular, la Universidad del Magdalena ha puesto a disposición herramientas tecnológicas de comunicación; capacitaciones en el uso de las TICs; asesorías psicológicas y de hábito saludable; charlas de cuidado ante la COVID-19: jornadas culturales y literarias virtuales y de seguimiento y auspicio a las actividades de docencia. La universidad también ha venido auspiciando activamente a sus estudiantes mediante la creación de un fondo de solidaridad, apoyo económico para estudiantes extranjeros, entrega de computadoras portátiles y datos de internet, periodos de gracia para matrículas, búsqueda de la gratuidad educativa para los estratos más bajos, entre otros.

La COVID-19 suscita algunas reflexiones y enseñanzas que vale la pena resaltar: i) La educación no se limita al aula física, esto es, las universidades presenciales deben reinventarse adoptando estrategias y tecnologías que democraticen el acceso a la educación.

ii) Reducir la brecha digital es crucial, es decir, buscar que más estudiantes accedan a internet $\mathrm{y}$ dispongan de computadoras o centros de cómputos comunitarios, lo que implica trabajo conjunto entre entidades estatales y empresas de telecomunicaciones.

iii) Capacitar y actualizar frecuentemente al personal docente y estudiantes en el uso de las TICs: creación de guías de desarrollo virtual, videoclases, asesorías - laboratorios virtuales, claustros docentes virtuales. Evaluar la posibilidad de asignación de algunas horas virtuales en plan de trabajo docente, que, sin afectar el carácter presencial de los programas de pregrado, mantenga vinculado al docente en el uso de herramientas nopresenciales.

iv) Involucrar a padres en el proceso pedagógico de los hijos, instruyéndolos en el manejo de las TICs.

v) Explorar las bondades de softwares de realidad virtual y aumentada ${ }^{13,15-17}$ para el desarrollo de actividades clínicas en pregrados de ciencias de la salud.

Tal vez ha llegado el momento para que docentes y directivos docentes de educación presencial cogiten acerca de la importancia e inclusión permanente de las tecnologías de la información y la comunicación en sus procesos pedagógicos, porque ha quedado claro que la vida siempre encuentra modos de promover cambios. Que la COVID-19 sea motivo para reflexionar sobre paradigmas arraigados en la educación presencial.

\section{REFERENCIAS BIBLIOGRÁFICAS}

1. Ozturk I. The Role of Education in Economic Development: A Theoretical Perspective. SSRN Electron J. 2011;33(1):39-47. Doi: http://dx.doi.org/10.2139/ssrn.1137541 
2. Bonilla-Mejía L, Sánchez-Jabba AM, Barón-Rivera JD, Bonilla-Mejía L, Galvis-Aponte LA, CepedaEmiliani $L$, et al. Educación y desarrollo regional en Colombia [Internet]. Primera Ed. Nomos, editor. Bogotá, Colombia: Banco de la República; 2014. p. 1$172 . \quad$ Disponible en: http://repositorio.banrep.gov.co/handle/20.500.12 134/9295

3. Valdés Pasarón $S$, Ocegueda Hernández JM, Romero Gómez A. La calidad de la educación y su relación con los niveles de crecimiento económico en México. Econ Desarro [Internet]. 2018;159(1):61$79 . \quad$ Disponible en: http://scielo.sld.cu/scielo.php?script=sci_arttext\&p id=S0252-85842018000100005

4. UNESCO. Educación [Internet]. [citado 5 de mayo de 2020]. p. 1-15. Disponible en: https://es.unesco.org/creativity/sites/creativity/file s/digital-library/cdis/Educacion.pdf

5. Canals C. Education and economic growth [Internet]. CaixaBank Research. 2017 [citado 5 de mayo de 2020]. p. 32-3. Disponible en: https://www.caixabankresearch.com/sites/default/ files/documents/IM_1705_32-33_D1_en.pdf

6. Valero A, Van Reenen J. The economic impact of universities: Evidence from across the globe. Econ Educ Rev. 2019;68(1):53-67. Doi: https://doi.org/10.1016/j.econedurev.2018.09.00

7. Yong, E, Nagles, N, Mejía, C Chaparro C. Evolución de la educación superior a distancia: desafíos y oportunidades para su gestión. Rev Virtual Univ Católica del Norte [revista en la Internet]. 2017 [citado 15 de mayo de 2020];(50):81-105. Disponible en:

http://revistavirtual.ucn.edu.co/index.php/Revista UCN/article/view/814/1332

8. Amato D, de Jesús Novales-Castro X. Utilidad para el aprendizaje de una modalidad educativa semipresencial en la carrera de Medicina. Investig en Educ Médica. 2014;3(11):147-54. Doi: https://doi.org/10.1016/S2007-5057(14)72741-3
9. Marco Antonio Márquez Gómez. Análisis de la oferta no presencial* de programas académicos de salud en Colombia [Internet]. 2017 [citado 6 de mayo de 2020]. Disponible en: http://congresomundial.unad.edu.co/images/pone ncias_y_conferencias/ponencias/dia 2_19 de mayo/Sala 6/Análisis de la oferta no presencial de programas académicos de salud en colombia.pdf

10. Díaz Sandoval. M. Una educación cada vez menos física. El Espectador [Internet]. 2018 [citado 15 de mayo de 2020]. Disponible en: https://www.elespectador.com/noticias/educacion /una-educacion-cada-vez-menos-fisica-articulo735695

11. Palacios Cruz M, Santos E, Velázquez Cervantes MA, León Juárez M. COVID-19, una emergencia de salud pública mundial. Rev Clínica Española. 2020;S0014-2565(20)30092-8.

Doi: https://doi.org/10.1016/j.rce.2020.03.001

12. Ministerio de Salud de Colombia. Reporte COVID-19 [Internet]. 2020 [citado 13 de mayo de 2020]. Disponible en: https://twitter.com/MinSaludCol/status/12603285 00109758466

13. Saavedra J. COVID-19 y Educación: Algunos desafíos y oportunidades [Internet]. Banco Mundial Blogs. 2020 [citado 6 de mayo de 2020]. Disponible en:

https://blogs.worldbank.org/es/education/educati onal-challenges-and-opportunities-covid-19-

pandemic

14. UNESCO. Consecuencias negativas del cierre de las escuelas [Internet]. 2020 [citado 6 de mayo de 2020]. Disponible en: https://es.unesco.org/covid19/educationresponse/ consecuencias

15. Garvía Vega L. COVID-19: Nueve razones por las que el mundo no volverá a ser el mismo. The Conversation [Internet]. 2020 [citado 15 de mayo de 2020]. p. 1. Disponible en: https://theconversation.com/covid-19-nueverazones-por-las-que-el-mundo-no-volvera-a-ser-elmismo-136155 
16. Pantelidis $\mathrm{P}$, Chorti A, Papagiouvanni I, Paparoidamis G, Drosos C, Panagiotakopoulos T, et al. Virtual and Augmented Reality in Medical Education. En: Medical and Surgical Education - Past, Present and Future. 2018. p. 77-97.

17. Pires LA, Serpa YR, Rodrigues MAF. SimImplanto - A Virtual Dental Implant Training Simulator. En: Proceedings - 18th Symposium on Virtual and Augmented Reality. 2016. p. 193-7. 